\title{
Estudio del comportamiento de sensores de gas basados en resonadores de cristal de cuarzo para altas frecuencias
}

\section{Study of the behavior of gas sensors based on quartz crystal resonators for high frequencies}

\author{
JIMÉNEZ-ARELLANO, Juan Jesús $\dagger^{*}$, MUÑOZ-MATA, José Lorenzo, MUÑOZ-AGUIRRE, Severino \\ y TORRES-MÉNDEZ, Sergio
}

Universidad Tecnológica de Puebla

Benemérita Universidad Autónoma de Puebla

Instituto Tecnológico de Puebla

\author{
ID $1^{\text {er }}$ Autor: Juan Jesús, Jiménez-Arellano / ORC ID: 0000-0002-0279-1999, CVU CONACYT ID: 177103 \\ ID $1^{\text {er }}$ Coautor: José Lorenzo, Muñoz-Mata / ORC ID: 0000-0001-7813-5579, CVU CONACYT ID: 177117 \\ ID $2^{\text {do }}$ Coautor: Severino, Muñoz-Aguirre / ORC ID: 0000-0002-1822-1464, CVU CONACYT ID: 121589 \\ ID $3^{\text {er }}$ Coautor: Sergio, Orres-Méndez / ORC ID: 0000-0001-7399-784X, CVU CONACYT ID: 177116
}

DOI: $10.35429 /$ JSI.2019.10.3.29.37

Recibido 16 de Abril, 2019; Aceptado 20 Junio, 2019

\section{Resumen}

Los sensores de microbalanza de cristal de cuarzo (QCM) se han empleado frecuentemente como dispositivos de micro-pesaje, puesto que han demostrado ser altamente sensibles debido a corrimientos en su frecuencia de resonancia a causa de incrementos en la masa adherida sobre su superficie. Este tipo de dispositivos son utilizados normalmente como arreglos de sensores para los sistemas conocidos como Narices Electrónicas, para la detección y análisis de gases, fluidos, y compuestos biológicos entre otros. La sensibilidad de los sensores QCM es directamente proporcional a la frecuencia de resonancia del cristal. Por lo que incrementar la sensibilidad, implica usar cristales de altas frecuencias por arriba de los 20 MHz. Se han fabricado cristales en dos modalidades: fundamental y sobretono, siendo los últimos empleados para frecuencias entre los $30 \mathrm{MHz}$ y $200 \mathrm{MHz}$. En este trabajo se muestran argumentos teóricos y resultados experimentales que describen las características y diferencias en el desempeño de sensores a $30 \mathrm{MHz}$ en ambas modalidades, además de otras consideraciones en el comportamiento de estos dispositivos que no se pueden observar desde el punto de vista analítico, pero si desde el punto de vista experimental.

Sensor QCM de Alta Frecuencia, Sobretono, Nariz Electrónica

\begin{abstract}
Quartz crystal microbalance (QCM) sensors have been frequently used as micro-weighing devices, since they have demonstrated to be highly sensitive due to the frequency shifts ought to mass- increments stuck on the sensing film surface of the sensor. This kind of devices are normally used as arrays of sensors in systems known as Electronic Noses, for the detection and analysis of gases, fluids, and biological compounds among others. The sensitivity of the QCM sensors is directly proportional to the resonance frequency of the crystal. Therefore, increasing sensitivity implies using high frequency crystals above $20 \mathrm{MHz}$. Quartz crystals have been manufactured in two modalities: fundamental and overtone, the latter being used for frequencies between 30 $\mathrm{MHz}$ and $200 \mathrm{MHz}$. In this work, we present theoretical arguments as well as experimental results that describe the characteristics and differences in the performance of 30 $\mathrm{MHz}$ sensors in both modalities, in addition to other considerations in the behavior of these devices that cannot be observed from the analytical point of view, but from the point of experimental view.
\end{abstract}

High-Frequency QCM Sensors, Overtone, Electronic Nose

Citación: JIMÉNEZ-ARELLANO, Juan Jesús, MUÑOZ-MATA, José Lorenzo, MUÑOZ-AGUIRRE, Severino y TORRESMÉNDEZ, Sergio. Estudio del comportamiento de sensores de gas basados en resonadores de cristal de cuarzo para altas frecuencias. Revista de Innovación Sistemática 2019. 3-10:29-37

*Correspondencia al Autor (Correo electrónico: juan.jimenez@utpuebla.edu.mx)

$\dagger$ Investigador contribuyendo como primer Autor. 


\section{Introducción}

Las narices electrónicas (NE) son complejos sistemas electrónicos capaces de detectar y analizar componentes volátiles. Existen muchos sensores comerciales para gases, y uno de los más empleados son los del tipo microbalanza de cristal de cuarzo o QCM (Quartz Crystal Microbalance por sus siglas en inglés) tipificados por su frecuencia resonante de operación (Gardner, Bartlett 1999). La respuesta típica de estos sensores ante un particular componente volátil, genera un decremento significativo en su frecuencia resonante; comportamiento descrito a través de un estudio hecho por Günter Sauerbrey en 1959. Las relaciones cuantitativas del estudio muestran que, si se incrementa la frecuencia resonante, mayor será el decremento significativo en su frecuencia; dicho en otras palabras, se incrementa la sensibilidad del sensor y por ende la de la nariz electrónica.

La sensibilidad de cualquier sensor y especialmente para aquellos empleados en una nariz electrónica, es un parámetro sumamente importante; pues de ello depende que tan capaz el sistema pueda detectar bajos niveles de concentración de el o los componentes volátiles. Niveles de concentración tan bajos que pueden ser indetectables para ciertos sensores y mucho más para una nariz humana.

Existen muchas razones por las cuales es importante tener una nariz electrónica con alta sensibilidad. En muchas de las industrias, muy a menudo se presentan situaciones donde las concentraciones de ciertas sustancias pueden alcanzar niveles críticos y riesgosos tanto para la salud como para la seguridad de las personas ( $\mathrm{Pi}$ Guey, Tsao-Yun 2017). En domótica para monitorear niveles muy bajos de gas natural, LP y otras sustancias de uso común. En la industria farmacéutica y biotecnología para la detección de enfermedades a través del estudio de reacciones químicas de ciertos agentes biológicos (Gardner, Bartlett 1994). A la fecha ya se empiezan a dar aplicaciones en la industria de los electrodomésticos, cosméticos y automotriz.
Muchos han sido los trabajos acerca de los sensores de gas del tipo QCM; empleando para su implementación, resonadores de cristal de cuarzo cuyas frecuencias van desde $5 \mathrm{MHz}$ hasta $20 \mathrm{MHz}$ (Muñoz, Nakamoto and Moriizumi 1999); presentando algunos rasgos característicos en común: por un lado, los circuitos osciladores utilizados para estos sensores se centran en el empleo de compuertas lógicas del tipo TTL o CMOS (Nakamoto, Nakamura and Moriizumi 1996) y esto restringe su operación a las frecuencias límites de las compuertas (aproximadamente a $30 \mathrm{MHz}$ ).

Por otro lado, muchos trabajos emplean QCMs con resonadores que fueron fabricados en modo fundamental (Speller, Siraj, McCarter and Vaughan 2017) y comúnmente los fabricantes manufacturan resonadores en un margen entre $1.8 \mathrm{MHz}$ y $25 \mathrm{MHz}$ en este modo (International Crystal Manufacturing 2004).

Para frecuencias mayores; el incremento en la complejidad del proceso de fabricación y los costos, hace que los fabricantes sólo manufacturen resonadores de este tipo en pequeños lotes y para pedidos especiales (Stehrer, Schwödiauer, Graz, Pollheimer and Gruber 2010). A cambio de esto, se fabrican resonadores en modo de sobretono para contemplar frecuencias desde $16 \mathrm{MHz}$ hasta 200 $\mathrm{MHz}$ (International Crystal Manufacturing 2004). Utilizar resonadores de esta clase implica por un lado emplear componentes de altas frecuencias para los circuitos; y por el otro, se requieren modificaciones en los osciladores para que estos resonadores puedan trabajar a altas frecuencias, algo que no es tan inmediato y fácil de implementar (Alassi, Benammar, Dan Brett 2017).

El presente trabajo se enfoca en el tratamiento y aplicación de los sensores QCM creados con resonadores de altas frecuencias; se muestra un estudio teórico y experimental sobre sus rasgos característicos más importantes tanto de comportamiento como de desempeño, además de un comparativo en relación con aquellos sensores creados con resonadores de bajas frecuencias. El desarrollo del artículo se ha dividido en varias secciones: fundamentos teóricos acerca de los sensores QCM, circuito oscilador para altas frecuencias, implementación de sensores QCM para altas frecuencias, arreglo y desarrollo experimental, obtención y discusión de resultados, conclusiones y trabajo a futuro. 


\section{Fundamentos Teóricos Acerca de los Sensores de Microbalanza de Cristal de Cuarzo}

Los resonadores de cristal de cuarzo son dispositivos que rigen su funcionamiento a través de un fenómeno conocido como efecto piezoeléctrico; que en breves palabras se puede decir que bajo ciertas condiciones: a) un cristal sometido a un esfuerzo mecánico puede generar una diferencia de potencial en el extremo de sus terminales (efecto piezoeléctrico directo) o b) un cristal al que se le aplique una fuerza electromotriz variante en el tiempo genera un movimiento mecánico de desplazamiento en su estructura física traducido en oscilaciones permanentes y precisas (efecto piezoeléctrico inverso). Es debido a este fenómeno piezoeléctrico inverso que los cristales de cuarzo pueden ser utilizados como resonadores para la implementación de sensores microgravimétricos capaces de detectar masas muy pequeñas de varios ordenes de magnitud (Arnau 2004). Entre estos dispositivos destacan los sensores QCM, que se emplean para la detección de componentes volátiles, fluídicos y algunas otras propiedades físicas (Theisen, Martin 2004).

Günter Sauerbrey (Sauerbrey 1959) mostró que el comportamiento de los QCM, cuando son empleados para la detección de pequeñas cantidades de masa, puede ser cuantitativamente expresado como lo muestra la Ecuación 1.

$\Delta \mathrm{f}_{\mathrm{N}}=-\frac{2}{\sqrt{\rho_{\mathrm{q}} \mu_{\mathrm{q}}}} \frac{\Delta \mathrm{mf}_{\mathrm{N}}{ }^{2}}{\mathrm{~N} \cdot \mathrm{A}}$

Donde $\Delta \mathrm{f}_{\mathrm{N}}$ denota el cambio en la frecuencia resonante del sensor, el signo menos indica que el cambio es un decremento. $\Delta \mathrm{m}$ es la cantidad de masa agregada sobre la superficie efectiva del electrodo del resonador, $\mathrm{f}_{\mathrm{N}}$ es la frecuencia resonante del QCM, A es el área efectiva sobre el cual se deposita la masa excedente, $\rho_{\mathrm{q}}$ y $\mu_{\mathrm{q}}$ son respectivamente la densidad y el módulo de cizalla del cuarzo. $\mathrm{N}$ es un numero entero impar positivo que indica el modo resonante del sensor siendo 1 para el modo fundamental y para los demás valores el respectivo sobretono.

De la ecuación de Sauerbrey se puede observar que si se incrementa la frecuencia resonante del sensor $f_{N}$, se incrementa el valor $\operatorname{para} \Delta \mathrm{f}_{\mathrm{N}}$.
Más aún, mediante una manipulación algebraica se establece una relación entre las respuestas para un mismo QCM en sus modos fundamental y alguno de sus sobretonos, como lo indica la Ecuación 2.

$\Delta \mathrm{f}_{\mathrm{N}}=\mathrm{N} \cdot \Delta \mathrm{f}_{1}$

Existen algunos casos donde hay resonadores de cuarzo fabricados con la misma frecuencia, pero en diferentes modalidades, comúnmente para el caso de $30 \mathrm{MHz}$. De manera general, si dos QCM con resonadores fabricados en la misma frecuencia: uno en modo fundamental y otro en $\mathrm{N}$ sobretono; y con la misma masa adicional $\Delta \mathrm{m}$ en sus electrodos, entonces se cumple la relación que muestra la Ecuación 3.

$\Delta \mathrm{f}_{1}=\mathrm{N} \cdot \Delta \mathrm{f}_{\mathrm{N}}$

Dicho de otra manera; QCMs con la misma frecuencia nominal, el sensor con resonador en modo fundamental es más sensible en un factor de $\mathrm{N}$ con respecto a su homólogo en modo de N sobretono. Desafortunadamente para los fabricantes es muy difícil y poco costeable fabricar exclusivamente resonadores de cuarzo en modo fundamental; pues entre más alta sea la frecuencia, más delgado es el espesor del cristal en un factor de $\mathrm{N}$ como lo muestran las dos relaciones en la Ecuación 4.

$\mathrm{l}_{1}=\frac{\mathrm{v}_{\mathrm{O}}}{2 \mathrm{f}_{1}}, \quad \mathrm{l}_{\mathrm{N}}=\frac{\mathrm{l}_{1}}{\mathrm{~N}}$

Donde $f_{1}$ es la frecuencia fundamental del resonador, $\mathrm{v}_{\mathrm{o}}$ es la velocidad de propagación de onda y $l_{\mathrm{N}}$ es el espesor del cristal de cuarzo.

\section{Circuito Oscilador para Altas Frecuencias}

Muchos circuitos osciladores se han presentado en la literatura tanto de baja como de alta frecuencia que emplean resonadores de cuarzo. Se pueden encontrar osciladores empleando compuertas lógicas, filtros, amplificadores operacionales, arreglo de transistores, combinaciones de estos y demás; donde la mayoría de ellos se han diseñado con un único propósito: ser señales de reloj para los sistemas digitales (Atmel Corporation 2004).

La situación cambia cuando el circuito oscilador forma parte de un sensor lo que implica un diseño y consideraciones técnicas más rigurosos.

JIMÉNEZ-ARELLANO, Juan Jesús, MUÑOZ-MATA, José Lorenzo, MUÑOZ-AGUIRRE, Severino y TORRES-MÉNDEZ, Sergio. Estudio del comportamiento de sensores de gas basados en resonadores de cristal de cuarzo para altas frecuencias. Revista de Innovación Sistemática 2019 
Factores como ruido e interferencia de cualquier naturaleza, afecta significativamente el desempeño del oscilador. El circuito de la Figura 1 muestra el oscilador empleado en este trabajo.

Las características de este oscilador son: un buen ancho de banda para altas frecuencias, configuración de seguidor de emisor por lo que su ganancia en voltaje es aproximadamente 1, una impedancia de entrada muy alta de tal manera que la demanda de carga para el QCM es muy pequeña, la impedancia de salida es muy baja lo que permite que prácticamente toda la carga del oscilador pase integra a la siguiente etapa del sistema.

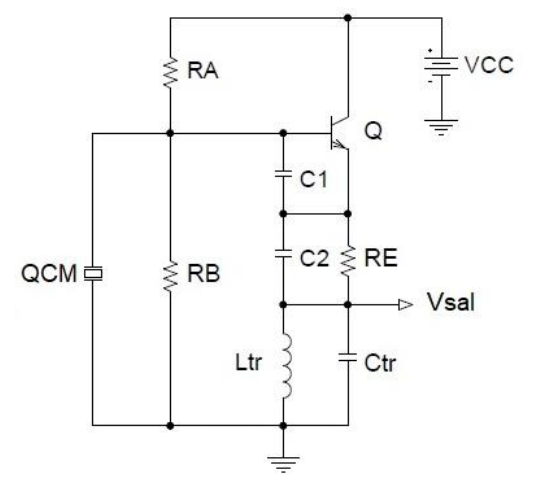

Figura 1 Circuito oscilador para QCM de alta frecuencia Fuente: Elaboración Propia

El circuito se puede ajustar para oscilar en una frecuencia específica entre $4 \mathrm{MHz}$ y 100 $\mathrm{MHz}$. Todas estas características hacen que este oscilador sea útil para los propósitos de este trabajo. En el Gráfico 1 se muestra una simulación en PSpice para un resonador de 30 $\mathrm{MHz}$ en modalidad de 3er sobretono notándose claramente los estados transitorio y permanente de la señal en el domino del tiempo. El Gráfico 2 ilustra el espectro en frecuencia de la simulación en PSpice de la señal en el tiempo que genera el oscilador; siendo poco notoria la presencia de los armónicos de la señal con respecto a la frecuencia principal.

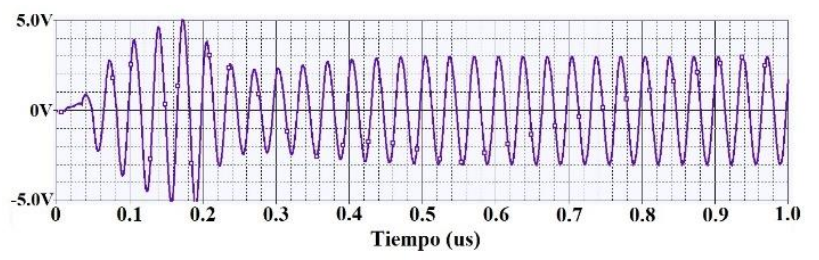

Gráfico 1 Simulación en PSpice de la señal en el domino del tiempo para un resonador de $30 \mathrm{MHz}$ en modo de $3 \mathrm{er}$ sobretono

Fuente: Elaboración Propia

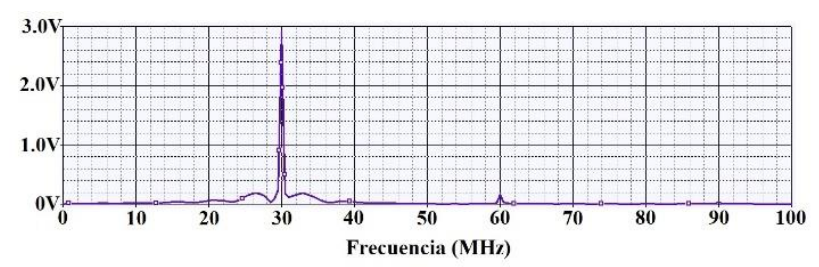

Gráfico 2 Simulación en PSpice del espectro en frecuencia de la señal del oscilador para un resonador de $30 \mathrm{MHz}$ en modo de 3er sobretono

Fuente: Elaboración Propia

\section{Implementación de Sensores QCM para Altas Frecuencias}

Un sensor QCM se implementa a partir de un resonador de cuarzo cuya protección metálica, que proviene de fábrica, se retira cuidadosamente dejando expuesto el cristal y los electrodos adjuntos. En este trabajo se han empleado resonadores del tipo HC49U con corte del cristal AT debido a disponibilidad, su excelente y constante comportamiento ante variaciones de temperatura, así como su bajo costo (International Crystal Manufacturing 2004); como lo muestra la Figura 2.

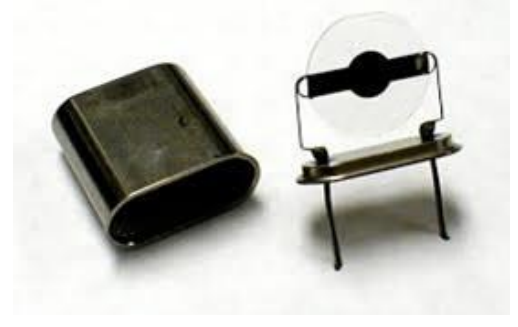

Figura 2 Resonador de cuarzo del tipo HC49U sin su protección de fábrica

Fuente: Elaboración Propia

Después de la remoción de su protección, el cristal pasa por un proceso de limpieza de algunos minutos a través de acetona y exposición en una cámara de Ultravioleta-Ozono para remover cualquier impureza que haya estado presente en la superficie de sus electrodos. Posterior a este proceso; el resonador está listo para el depósito, sobre sus electrodos, de una delgada película hecha con un material que es químicamente sensible al componente volátil a detectar.

El depósito es hecho a través del método de casting que básicamente consiste en fijar el QCM, tomar con una pipeta graduada, una pequeña muestra de la solución hecha para la película sensible y depositar en el centro de ambos electrodos la gota-muestra del material, como lo ilustra la Figura 3. 


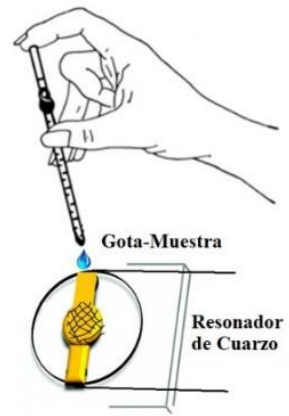

Figura 3 Implementación de un QCM por medio del método de casting

Fuente: Elaboración Propia

Una vez realizado el depósito de la película, se toma un tiempo de espera para el secado del material colocando los sensores dentro de una cámara desecadora a una temperatura de $25^{\circ} \mathrm{C}$ y $10 \%$ de humedad constantes. Posterior al proceso de secado, el sensor QCM está listo para la etapa de prueba.

Un parámetro muy crítico para un QCM es el espesor de la película sensible depositada, como lo muestra la Figura 4. Poder hacer una medición directa del espesor sobre el dispositivo es muy complicado. Por lo tanto, es por medio de la ecuación de Sauerbrey, a través de una manipulación algebraica, que se puede estimar este parámetro de manera indirecta como lo muestra la Ecuación 5.

$\mathrm{l}_{\mathrm{ps}(\mathrm{N})}=\frac{\mathrm{N} \cdot \Delta \mathrm{f}_{\mathrm{N}} \sqrt{\rho_{\mathrm{q}} \mu_{\mathrm{q}}}}{2 \rho_{\mathrm{ps}} \mathrm{f}_{\mathrm{N}}{ }^{2}}$

Donde $\mathrm{l}_{\mathrm{ps}(\mathrm{N})}$ es el espesor estimado de la película sensible, $\rho_{\mathrm{ps}}$ es la densidad del material de la película y los demás parámetros ya se mencionaron en la sección 1.

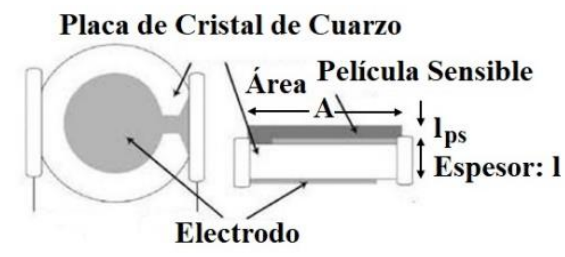

Figura 4 Vista de la implementación y parámetros de un QCM

Fuente: Elaboración Propia

Es de tomarse en consideración, que la cantidad del material depositado para la película sensible es crítica, pues un exceso hará que el QCM no pueda oscilar y no cumplir su propósito.
Dado el caso, es posible en su mayoría, poder remover la película y realizar un proceso de limpieza como se describió en esta sección, con el riesgo de quebrarse el cristal y en definitiva ya no servir el dispositivo.

Adjunto a lo anterior, las cantidades de material sensible depositadas para los QCMs de bajas frecuencias no son las adecuadas para los de alta frecuencia; por un lado, los cristales de los resonadores en modo fundamental son más pequeños y más delgados; y por el otro, el hecho de que un QCM oscile en su modo fundamental no significa que pueda oscilar para todos sus demás sobretonos. En definitiva, los tratamientos pueden ser semejantes en unos aspectos, pero totalmente diferentes en otros.

\section{Arreglo y Desarrollo Experimental}

El arreglo experimental para este estudio se muestra en la Figura 5. El sensor QCM es colocado dentro de una cámara hermética de teflón inmersa en un baño térmico para mantener las condiciones de humedad y temperatura constantes.

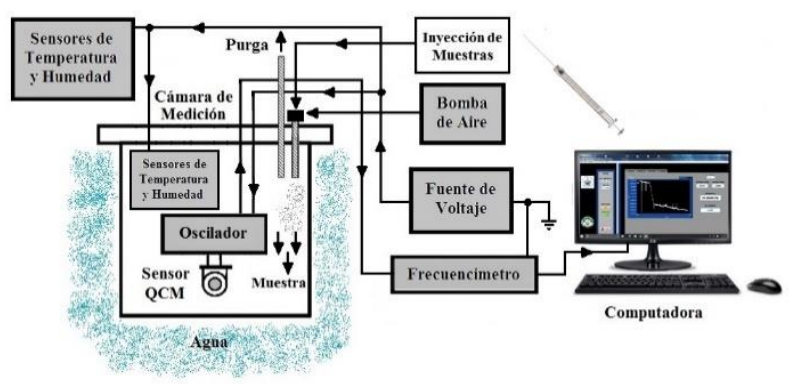

Figura 5 Arreglo experimental para el estudio del desempeño de sensores QCM de altas frecuencias Fuente: Elaboración Propia

El desarrollo experimental se lleva a cabo con 6 resonadores de cuarzo del tipo HC49U de $30 \mathrm{MHz}: 3$ en modo fundamental y 3 en modo de 3er sobretono. El componente volátil a detectar es etanol y el material que se emplea para la película sensible es una solución de etil-celulosa con cloroformo. La selección de la etil-celulosa es debido a que presenta una muy buena afinidad química al etanol para el proceso de adsorción de sus moléculas. La solución presenta una concentración de $2 \mathrm{mg} / \mathrm{ml}$, un nivel bajo debido a que un exceso de material hará que el QCM no pueda oscilar. 
La primera prueba que se realiza a los sensores, es la estimación del espesor de la película $\mathrm{l}_{\mathrm{ps}(\mathrm{N})}$ a través de la Ecuación 5. Una vez removidas las protecciones de los resonadores, se mide la frecuencia del dispositivo antes y después del depósito de la película, la diferencia de estas mediciones proporciona el valor para $\Delta \mathrm{f}_{\mathrm{N}}$. Mediante el método de casting y lo más cercano posible al centro de los electrodos de los 6 resonadores, se hace el depósito de una gota de $0.2 \mu l$ de la solución para la película y se espera un tiempo de alrededor de 1 hora para que seque totalmente la solución.

La segunda prueba es la detección del componente volátil. Una vez que el sensor alcanza una línea base estable en su frecuencia resonante, se hace la inyección de la muestra del componente. Inmediatamente, la muestra se evapora y sus moléculas empiezan a tener contacto con la película sensible; un proceso de adsorción de las moléculas se lleva a cabo, lo que incrementa la masa en los electrodos del cristal $y$ un decremento en su frecuencia resonante se ve reflejado. El Gráfico 3 ilustra la descripción anterior y muestra la respuesta típica de los sensores QCM. El carácter exponencial de la respuesta da una idea de cómo es el proceso de adsorción; posterior a este, una nueva línea base aparece indicando que ha terminado la adsorción. El proceso anterior se repite de manera consecutiva por lo menos tres veces para observar que tan lineal es el comportamiento de las respuestas de estos sensores.

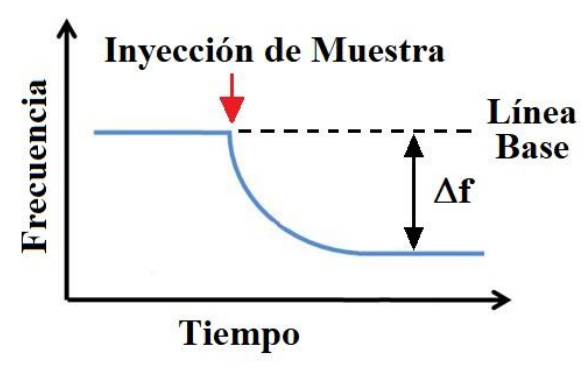

Gráfico 3 Respuesta típica de un sensor QCM Fuente: Elaboración Propia

La prueba culmina con la inyección de aire seco dentro de la cámara de medición para remover todo rastro del componente volátil y regresar al sensor a sus condiciones iniciales. El oscilador está conectado a un frecuencímetro de alta resolución capaz de detectar variaciones de hasta $1 \mathrm{~Hz}$ y una interface de usuario gráfica (GUI) muestra y registra el comportamiento del sensor durante las pruebas.

\section{Obtención y Discusión de Resultados}

Los resultados obtenidos en las mediciones de las frecuencias de los dispositivos antes y después del depósito de la película sensible se muestran en la Tabla 1.

\begin{tabular}{|c|c|c|c|c|}
\hline $\begin{array}{l}\text { Modo de } \\
\text { Operación }\end{array}$ & $\begin{array}{c}\text { Frecuencia } \\
\text { sin Película } \\
(\mathrm{MHz})\end{array}$ & $\begin{array}{c}\text { Frecuencia } \\
\text { con Película } \\
\text { (MHz) }\end{array}$ & $\begin{array}{c}\Delta \mathbf{f} \\
(\mathbf{k H z})\end{array}$ & $\begin{array}{c}\mathrm{l}_{\mathrm{ps}} \\
(\boldsymbol{\mu \mathrm { m }})\end{array}$ \\
\hline Fundamental & 29.99238 & 29.93750 & 54.88 & 0.24 \\
\hline Fundamental & 29.99233 & 29.96501 & 27.32 & 0.12 \\
\hline Fundamental & 29.99220 & 29.91513 & 77.07 & 0.33 \\
\hline 3er Sobretono & 29.99981 & 29.99001 & 9.81 & 0.13 \\
\hline 3er Sobretono & 29.99973 & 29.98154 & 17.67 & 0.23 \\
\hline 3er Sobretono & 30.00000 & 29.99216 & 7.84 & 0.10 \\
\hline
\end{tabular}

Tabla 1 Caracterización de los QCM de $30 \mathrm{MHz}$ antes y después del depósito de la película sensible

Fuente: Elaboración Propia

El objetivo de estimar cada uno de los espesores es determinar que par de sensores (fundamental y sobretono) tienen sus valores del espesor $\mathrm{l}_{\mathrm{ps}(\mathrm{N})}$ lo más cercano posible y ver el cumplimiento que establece la Ecuación 3. La Tabla 2 muestra los pares de estos sensores que cumplen el criterio.

\begin{tabular}{|l|c|r|r|r|}
\hline $\begin{array}{c}\text { Modo de } \\
\text { Operación }\end{array}$ & $\begin{array}{c}\text { Frecuencia } \\
\text { sin Película } \\
\text { (MHz) }\end{array}$ & $\begin{array}{c}\text { Frecuencia } \\
\text { con Película } \\
(\mathbf{M H z})\end{array}$ & \multicolumn{1}{c|}{$\begin{array}{c}\Delta \mathbf{f} \\
(\mathbf{k H z})\end{array}$} & $\begin{array}{c}\mathrm{l}_{\mathbf{p s}} \\
(\boldsymbol{\mu m})\end{array}$ \\
\hline Fundamental & 29.99233 & 29.96501 & 27.32 & 0.12 \\
\hline 3er Sobretono & 29.99981 & 29.99001 & 9.81 & 0.13 \\
\hline Fundamental & 29.99238 & 29.93750 & 54.88 & 0.24 \\
\hline 3er Sobretono & 29.99973 & 29.98154 & 17.67 & 0.23 \\
\hline
\end{tabular}

Tabla 2 Pares de sensores QCM con espesores de película sensible aproximados

Fuente: Elaboración Propia

De acuerdo a la Ecuación 5, el valor para $\Delta \mathrm{f}_{1} / \Delta \mathrm{f}_{3}=3$ y la Tabla 3 muestra el comparativo.

\begin{tabular}{|c|c|c|c|c|}
\hline $\begin{array}{c}\text { Modo de } \\
\text { Operación }\end{array}$ & $\underset{(\mu \mathrm{m})}{\mathrm{l}_{\mathrm{ps}}}$ & $\begin{array}{c}\Delta \mathbf{f} \\
(\mathbf{k H z})\end{array}$ & $\frac{\Delta f_{1}}{\Delta f_{3}}$ & $\frac{\Delta f_{1}}{\Delta f_{3}}$ Normalizado \\
\hline Fundamental & 0.12 & 27.32 & \multirow{2}{*}{2.78} & \multirow{2}{*}{3.02} \\
\hline 3er Sobretono & 0.13 & 9.81 & & \\
\hline Fundamental & 0.24 & 54.88 & \multirow{2}{*}{3.10} & \multirow{2}{*}{2.98} \\
\hline 3er Sobretono & 0.23 & 18.19 & & \\
\hline
\end{tabular}

Tabla 3 Comparativo entre QCMs de $30 \mathrm{MHz}$ en modo fundamental y de 3 er sobretono

Fuente: Elaboración Propia

Para la prueba del compuesto volátil se emplea una serie de 3 inyecciones de $5 \mu \mathrm{l}$ de etanol. Los Gráficos 4 y 5 muestran las respuestas obtenidas para los dos pares de sensores QCM de la Tabla 3. Las flechas rojas muestran el instante de la inyección acumulativa de la muestra de $5 \mu 1$ de etanol. 

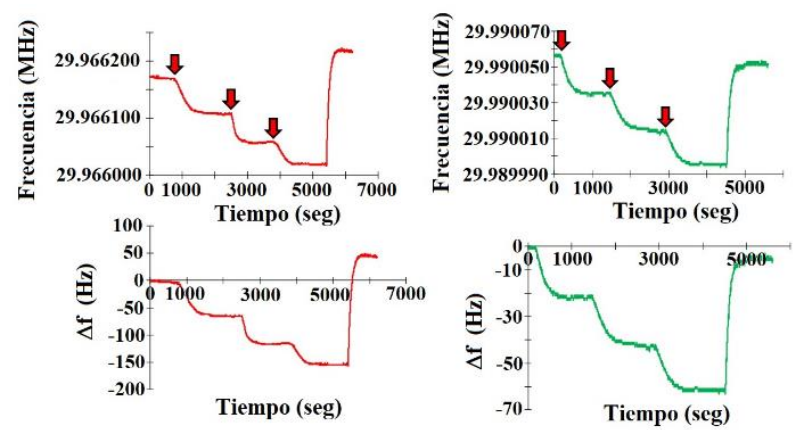

Gráfico 4 Respuesta de los sensores QCM de $30 \mathrm{MHz}$ de espesor aproximadamente $0.12 \mu \mathrm{m}$ ante una serie de 3 muestras de etanol de $5 \mu 1$ cada una. Lado izquierdo en fundamental, lado derecho en 3er sobretono

Fuente: Elaboración Propia
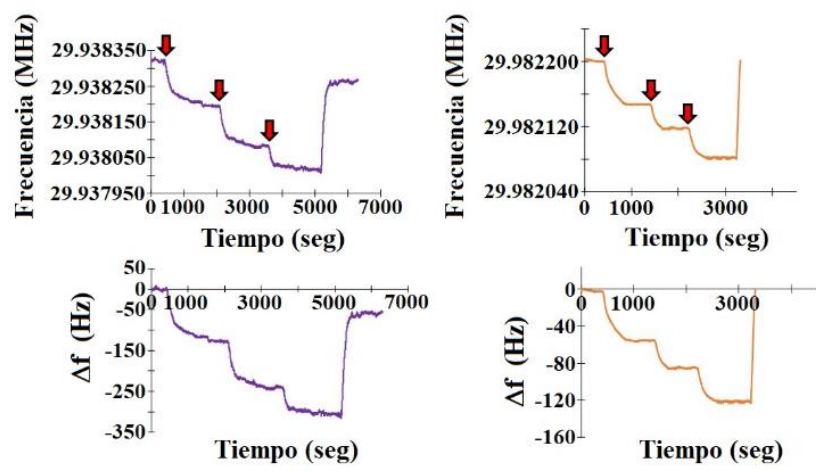

Gráfico 5 Respuesta de los sensores QCM de $30 \mathrm{MHz}$ de espesor aproximadamente $0.24 \mu \mathrm{m}$ ante una serie de 3 muestras de etanol de $5 \mu 1$ cada una. Lado izquierdo en fundamental, lado derecho en 3er sobretono

Fuente: Elaboración Propia

Los Gráficos 6 y 7 muestran las curvas de correlación de ajuste lineal para los puntos de dispersión de cada línea base conforme a las respuestas de los Gráficos 4 y 5 en función de la concentración de etanol (ppm) de cada muestra de $5 \mu \mathrm{l}$ de etanol acumulativa. Los cocientes entre las pendientes de ambos ajustes lineales $\mathrm{y}_{1} / \mathrm{y}_{3}=0.0333 / 0.0130 \approx 2.56$ para el primer par y $\mathrm{y}_{1} / \mathrm{y}_{3}=0.0668 / 0.0261 \approx 2.56$ para el segundo par marcan una tendencia hacia el valor 3 , como lo establece la Ecuación 5.

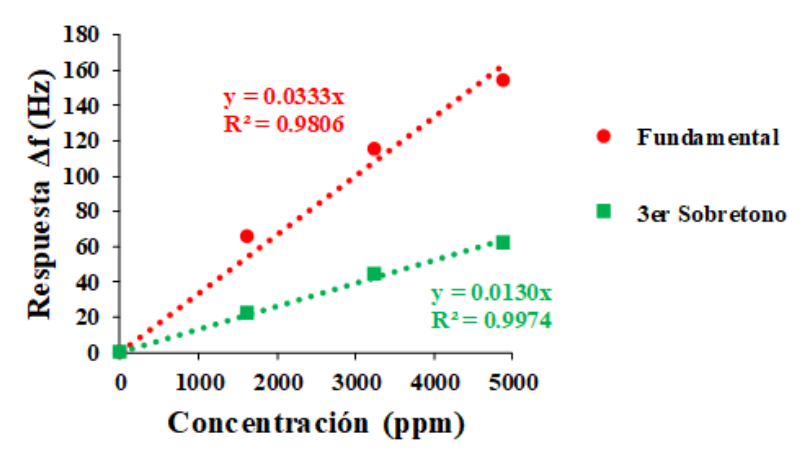

Gráfico 6 Curvas de correlación de ajuste lineal para las respuestas de los QCM de espesor aproximadamente 0.13 $\mu \mathrm{m}$ en función de la concentración de etanol de las muestras

Fuente: Elaboración Propia

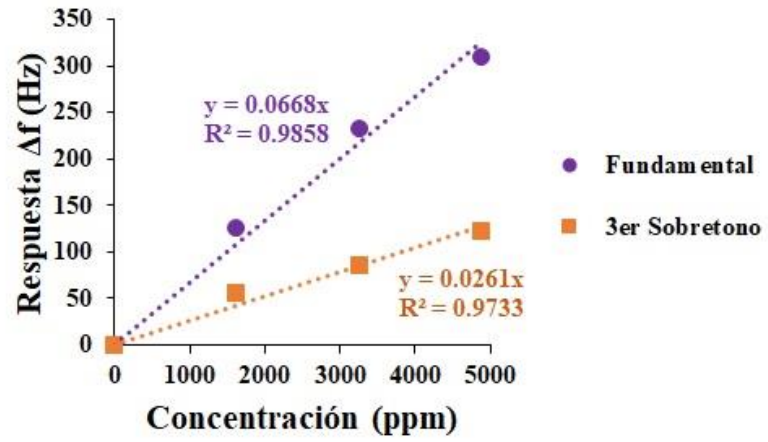

Gráfico 7 Curvas de correlación de ajuste lineal para las respuestas de los QCM de espesor aproximadamente 0.24 $\mu \mathrm{m}$ en función de la concentración de etanol de las muestras

Fuente: Elaboración Propia

Otra observación que es interesante marcar, es la comparación entre los espesores de ambos grupos.

$\frac{\mathrm{l}_{\mathrm{ps}(2 \mathrm{do} \text { Grupo })}}{\mathrm{l}_{\mathrm{ps}(1 \mathrm{er} \mathrm{Grupo})}}=\frac{0.24 \mu \mathrm{m}}{0.12 \mu \mathrm{m}}=2$

Y esta proporción también se ve reflejada si se comparan las pendientes de las curvas de correlación entre los correspondientes modos.

$\frac{\mathrm{y}_{\text {fund (2do Grupo) }}}{\mathrm{y}_{\text {fund (1er Grupo })}}=\frac{0.0668}{0.0333}=2$
$\frac{\mathrm{y}_{3 \operatorname{er~} S t(2 \text { do Grupo })}}{\mathrm{y}_{3 \operatorname{er~} \operatorname{St}(1 \mathrm{er} \mathrm{Grupo)})}}=\frac{0.0261}{0.0130}=2$

El comportamiento $\Delta$ fde los sensores QCM es directamente proporcional al espesor de la película sensible $\mathrm{l}_{\mathrm{ps}}$. Las variaciones entre los resultados teóricos y experimentales en el desempeño de los QCM se ven afectados por factores como la humedad y la temperatura; ambos factores tienden a disminuir tanto el cambio en la frecuencia $\Delta \mathrm{f}_{\mathrm{N}}$ como el tiempo de respuesta. Cualitativamente, las respuestas generadas por los QCM en modo de sobretono presentan menos fluctuaciones.

\section{Agradecimientos}

Se agradecen las facilidades otorgadas para la realización de este trabajo de investigación a la Universidad Tecnológica de Puebla (UTP). 


\section{Conclusiones}

Se puede establecer cuantitativamente una relación para el desempeño entre las respuestas de los sensores QCM en la modalidad de fundamental y sobretono (Ecuaciones 2, 3,5, 6, 7 y 8). Es muy importante tener un circuito oscilador de altas prestaciones en su diseño y desempeño pues si no cumple estas características, se verá reflejado un bajo desempeño de los QCM, principalmente por el rango de frecuencias que maneja y el ruido inherente al sistema completo.

La técnica de casting para la implementación de los QCM no es tan sofisticada, pero después de realizar varios depósitos se puede depurar y mejorarla. Los resultados obtenidos para la caracterización de los QCM antes y después del depósito de la película sensible dan muestra que no están muy lejos de los resultados esperados analíticamente.

Los resultados experimentales confirman que las respuestas obtenidas por los sensores ante la exposición de la serie de muestras acumulativas de etanol poseen un comportamiento lineal. Los parámetros de las curvas de correlación confirman que los QCM de mismas frecuencias en modo fundamental son 3 veces más sensibles que aquellos en modo de 3er sobretono. También es un hecho que si se desea aumentar la sensibilidad de los sensores se requieren resonadores en la modalidad de sobretonos. Finalmente se pudo comparar que los QCM de $30 \mathrm{MHz}$ presentan una mayor respuesta ante el etanol con respecto a un QCM de $12 \mathrm{MHz}$ en las mismas condiciones de prueba.

\section{Trabajo a futuro}

Siguiendo esta misma línea de trabajo, se está ya contemplando el análisis y desarrollo experimental para emplear resonadores de 40 $\mathrm{MHz}$ y $50 \mathrm{MHz}$ en 3er sobretono y ver que rasgos experimentales se pueden obtener. También se está contemplando implementar un arreglo de sensores QCM y ver su desempeño como un conjunto.

\section{Referencias}

Abdulrahman Alassi, Mohieddine Benammar, Dan Brett (2017). Quartz Crystal Microbalance Electronic Interfacing Systems: A Review. Sensors 2017172799.

Arnau A. (2004). Piezoelectric Transducers and Applications. Universidad Politécnica de Valencia, Spain. Springer-Verlag Berlin. J. W. Gardner, P. N. Bartlett (1999). Electronic Noses, ( $1^{\mathrm{a}}$ ed.). New York, USA: Bookcraft.

Atmel Corporation (2004). Analyzing The Behavior of an Oscillator and Ensuring Good Start-Up. Atmel 80C51 MCU's. Application Note.

Günter Sauerbrey (1959). Use of vibrating quartz for thin film weighing and microweighing. Zeitschrift Fuer Physik, 155, 206.

International Crystal Manufacturing (2004). Crystal Oscillator and Filter Products. Resistance Weld Crystal HC49U 13.

J. W. Gardner, P. N. Bartlett (1994). A Brief History of Electronic Noses, Sensors and Actuators B, 18, 210-211.

J. W. Gardner, P. N. Bartlett (1999). Electronic Noses, ( $1^{\mathrm{a}}$ ed.). New York, USA: Bookcraft.

Lisa A. Theisen, Stephen J. Martin (2004). A Model for the Quartz Crystal Microbalance Frequency Response to Wetting Characteristics of Corrugated Surfaces. Anal. Chem. 76, 796804.

Nakamoto T., Nakamura K., Moriizumi T. Study of Oscillator-Circuit Behavior for QCM Gas Sensor (1996). IEEE Ultrasonics Symposium, 351-354.

Pi-Guey Su, Tsao-Yun Chuang (2017). Simple and rapid differentiation of toxic gases using a quartz crystal microbalance sensor array coupled with principal component analysis. Sensors and Actuators A 263 1-7.

Severino Muñoz, Takamichi Nakamoto, Toyosaka Moriizumi (1999). Study of transient response of QCM odor/gas sensors coated with calixarene LB films. T. IEE Japan, Vol. 119-E, No. 8/9. 
Speller Nicholas C., Siraj Noureen, McCarter Kevin S., Vaughan Stephanie (2017). QCM Virtual Sensor Array: Vapor Identification and Molecular Weight Approximation. Sensor and Actuators B 246 952-960.

Stehrer B. P., Schwödiauer Bauer S., Graz I.M., Pollheimer P.D., Gruber H.J. (2010). High Frequency QCM based sensor system for sensitive detection of dissolved analytes. Procedia Engineering 5 835-837. 